\title{
Graphene steps into biomedicine
}

\author{
Biomedical applications for graphene are attracting interest from academics and industrial partners \\ aiming to develop next-generation medical devices and therapies.
}

April 2016 marks the beginning of a new phase of the Graphene Flagship ${ }^{1}$. This first phase of the Core Project - lasting for 3 years and supported by Horizon 2020, the EU Framework Programme for Research and Innovation (http://go.nature. com/CNOjo6) - consolidates some of the topics already explored during the ramp-up phase of this $€ 1$ billion research initiative, and further extends the technological and scientific remit by launching Work Packages focused on new applications (http:// go.nature.com/CQ1nIM). Among them, a new Biomedical Technologies Work Package will investigate whether $2 \mathrm{D}$ materials can be leveraged for the development of diagnostic or therapeutic tools for healthcare.

In keeping with the spirit of the Flagship, fundamental research in this area will need to attract the interest of industrial partners, who will have to provide part of the financial support for technological development, as well as assist with the clinical translation of the research output. Promoting these collaborations was the aim of a two-day Graphene Connect workshop (http://go.nature.com/y66MWr) held in February at the MINATEC centre, Grenoble, where scientists discussed with industry representatives how graphene might provide specific advantages for use in biomedical applications.

During his introductory talk, Kostas Kostarelos (University of Manchester, UK) - who leads this new Work Package together with Jose Garrido (Catalan Institute of Nanoscience and Nanotechnology, Barcelona, Spain) - articulated in very simple terms the first question that must be considered: "why graphene?" Indeed, although graphene possesses certain properties that are desirable for several high-tech applications, what technological requirements this and other $2 \mathrm{D}$ materials could address in biomedicine requires further investigation.

Implantable electronic devices - such as artificial retina implants or the bioelectronics interfaces envisioned by GlaxoSmithKline (a partner of the new Work Package) to treat diseases using electrical stimuli ${ }^{2}-$ must fulfil strict performance requirements to reduce patient discomfort. For instance, they must be small, flexible, stable in physiological conditions and able to provide

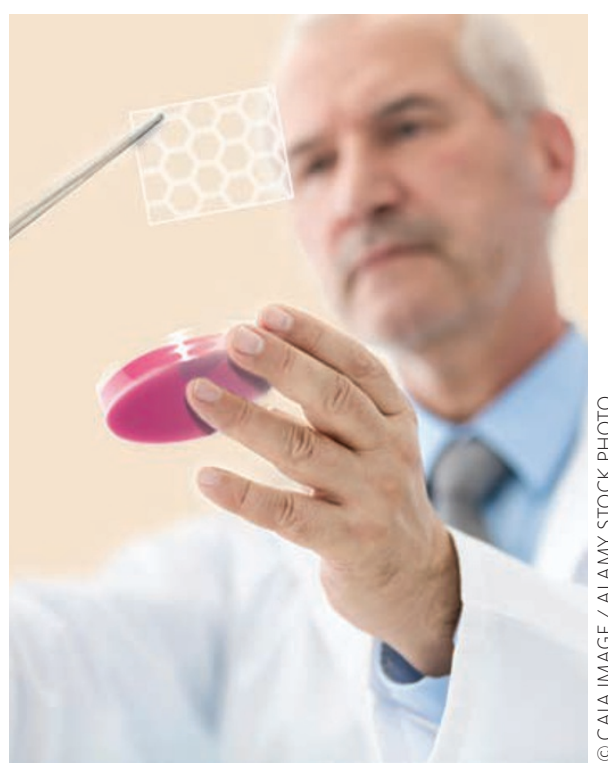

demonstration of its biocompatibility. It is not appropriate to make general statements on the behaviour of graphene, graphene oxide and other 2D materials when interacting with complex biological systems. Rather, specific properties such as flake size, defect density and surface chemistry dictate whether graphene is biologically inert or able to maintain therapeutic effectiveness without any kind of undesirable toxicity ${ }^{4}$. In future, it will therefore be particularly important to qualify any claims of graphene biocompatibility to account for these kinds of material characteristics. Only through this kind of detailed assessment can an optimal biological profile be achieved.

Graphene has been touted as the next big thing in several industries other than biomedicine, from energy generation and storage to optoelectronics and structural materials. However, investor enthusiasm may be tempered by the long commercialization times and high development costs reliable electrical performance for years. Although graphene possesses the structural and mechanical properties required by these devices, its stability and reliable operation in situ is less certain and will depend heavily on the quality and chemical reactivity of the specific materials used. Whether graphenebased interfaces can deliver electrical signals intense enough to stimulate the human nervous system, or outperform the sensors currently used to detect neuronal electrical activity, are also open questions that several groups, including that of Garrido, are trying to answer.

The application of graphene to assist drug delivery is another area of potential significance. Many drugs with beneficial therapeutic properties can also present detrimental side effects, limiting their clinical application. The use of nanomaterials to immobilize and deliver these drugs can assist with preventing undesirable effects. Also, complementary studies are exploring strategies to controllably release the immobilized drugs when the appropriate biological target is identified. The ease by which graphene can be chemically modified seemingly lends itself well to such immobilization and delivery applications ${ }^{3}$. What will likely be a much more significant hurdle is the requirement that any graphene-based drug delivery system should present a watertight that material-based innovations have historically experienced. As explained in the Commentary on page 487 of this issue by Elicia Maine and Purnesh Seegopaul (Simon Fraser University, Vancouver, Canada), the success of a new material in improving the performance of an end product also depends on the complementary adaptation of manufacturing processes or device designs, which unavoidably requires additional time for its industrial adoption. To avoid discouraging investors' interest, venture companies developing advanced materials should identify the most valuable commercial applications at an early stage and steer efforts in materials and manufacturing development accordingly.

Whether or not new diagnostic and therapeutic devices will be one of the graphene applications to be prioritized is an interesting question that both the Graphene Flagship and researchers worldwide are now trying to explore. The next few years will tell us how far graphene's steps into biomedicine can go.

\footnotetext{
References

1. Nature Mater. 12, 277 (2013).

2. Famm, K., Litt, B., Tracey, K. J., Boyden, E. S. \& Slaoui, M. Nature 496, 159-161 (2013).

3. Kuila, T. et al. Prog. Mater. Sci. 57, 1061-1105 (2012)

4. Bussy, C., Jasim, D., Lozano, N., Terry, D. \& Kostarelos, K. Nanoscale 7, 6432-6435 (2015).
} 